\title{
The application of statistic methods in commercial enterprises
}

\author{
Zhaoxu Liu \\ School of Business and Economics, Fayetteville State University, Fayetteville, NC 28301, US \\ Liuzx90@gmail.com
}

\begin{abstract}
In the process of the gradual maturity of the market economy in our country, many commercial enterprises have a lot of need for timely decision, at the same time they also often have to deal with a lot of randomness. Faced with a large amount of data and information, how to deal with, and how to make decisions? At this time, decision makers need to rely on statistical methods. Therefore, the statistical method plays an important role in the process of enterprise development. The author of this paper has discussed the application of statistic method and its related efficiency in Enterprises due to its important role in the development of the enterprises.
\end{abstract}

Keywords: statistic method; commercial enterprises.

\section{Introduction}

British scholar Wells said: "Statistical thinking method, as the ability to read and write, will become the necessary ability of the efficiency of citizens in the future." Britain, Japan, the United States, France, Sweden, Singapore, Canada and other countries have introduced the probability statistics to the primary and secondary school mathematics curriculum. They believe that knowledge of probability and statistics is more and more important in the information society, and it is an analytical tool that students who live in twenty-first Century must have a good grasp of.

For the business enterprises in the economic social, they will face a large number of random phenomena and the involved large amounts of data in their daily business process, how to deal with the data, and get some valuable information to make effective response? And For many uncertain factors in the enterprise management, how to carry on the analysis processing and then get the more reliable conclusion? Enterprise managers and operators only skillfully use the theory of probability and mathematical statistics to deal with this problem.

Statistical methods can be applied in all phases of the product life cycle, and more early application, more effective. Statistical methods can monitor the various stages of the product life cycle, judge the quality variation and timely alarm. If the enterprise can seriously implement the statistical techniques, rather than deal with the job, will bring benefits to the enterprise, so as to avoid futile.

\section{On Application Demand of Statistic Method in Enterprises}

The application of statistical method in enterprises has two premises: one is In the management system, it is necessary to clarify the status and guiding role of statistical methods, and the elements of statistical techniques must be used in the course of its operation; the second is the implementation of the program of statistical techniques must have maneuverability, rather than a prop. The application requirements of enterprise statistical methods are mainly in the following aspects:

(1) Combined with design and development control. Design quality ensures the product quality, and the design stage is the source of product quality. Improve the quality of design can make the product quality continuously meet its technical requirements, and to meet customer needs in order to produce leaps and innovation.

(2) Combined with production process control. On the application of statistical methods in the process of special process, the emphasis is key process parameters and quality indicators. Statistical methods are helpful for process improvement in the process of controlling and verifying process capability and product characteristics. The control chart can be used to monitor the production process; the hypothesis test can be used to verify the effectiveness of the process equipment. 
(3) Combined with inspection process control. The improvement of the quality of products is one of the effective marks of quality management, and the sampling method shows the effectiveness of the process control. The operation of enterprise quality management system emphasizes the improvement of quality, and the result of special analysis on the product process can be used as the input of process improvement and decision process to form a product failure report.

\section{The application of statistic methods in commercial enterprises}

\subsection{The application of probability theory in commercial enterprises}

Under the condition of market economy, the operation and sales of commercial enterprises are generally not determined by the subjective desires of the operators. It includes many uncontrollable specific issues: as in a unit of time, (such as days) how many customers patronize the mall; how many people that already entered the store have the real implementation of shopping behavior; how much each customer in the shopping activities buy, all of this is commodity problems and need to analyzed by means of probability method. Therefore, probability theory is widely used in commercial enterprises. Here are some of the typical problems faced by commercial enterprises to illustrate its application.

(1) Purchase problem. For example, every Thursday the shopping mall purchases goods to prepare for Friday, Saturday, Sunday, three days' sales. According to multi week statistics, the number of these three days of sales is independent of each other and the distribution of known. Then the problem that what's the values of three days of total sales of this random variable can be solved by the knowledge of probability theory.

(2) Resource allocation problem. For example, four salesmen in a counter in shopping mall, an average of an hour each salesperson only scale 15 minutes, so how many scale the shop need? We can use random variables obey the binomial distribution, the event of independence and small probability principle to solve the problem of resource allocation.

(3) Profit problem. For example, a commercial enterprise distribution of a certain commodity, the weekly purchase quantity of $\mathrm{X}$ and customer demand for the goods $\mathrm{Y}$ is two independent random variables, and is subject to the interval $[10,20]$ on the uniform distribution. Shop sold for every one unit of goods available profit of 1000 Yuan; if demand exceeds the amount of the purchase, the business enterprise can adjust the supply of goods from other commercial enterprises, then per unit of a commodity profit of 500 Yuan, the calculation of goods distribution obtained by the commodity weekly average profit, you need through the continuous calculation of binary random variable mathematical expectation to solve.

(4) Inventory problem. For example, enterprises need according to the Poisson distribution and probability theory knowledge to calculate the quantity of monthly store goods to ensure next month not out of stock and meanwhile not appear a large number of stocks.

(5) Decision problem. For example, a commercial enterprise has an advisory panel composed of nine people, each consultant with the correct views of 0.7 , now the enterprise seeks the each adviser individual advice of promote a strategy is feasible or not, and according to the views of the majority of people make decisions. This also can be solved by probability theory knowledge to calculate the probability of correct decisions to make.

\subsection{The application of mathematical statistics in commercial enterprises}

Mathematical statistics analysis method also has a wide range of applications in commercial enterprises. Therefore, here are some of the typical problems faced by commercial enterprises to illustrate its application.

(1) Product market share issue. For example, we can use the parameter interval estimation theory of mathematical statistics to process data, judge a product's market share in a certain confidence interval.

(2) The significance of the effect of adjustment measures. For example, in order to increase sales, a supermarket adjusts the sales, management and so on, after the adjustment, statistic the 9 day of 
random spot checks of daily sales, the average daily sales are 60 Yuan. According to statistics, before the adjustment, the average daily sales are 52 million Yuan, assumed daily sales follows a normal distribution. So whether the effect of adjustment measures is significant? Not intuitively think measures significantly just because after adjustment the sales reached 60 million Yuan, but by hypothesis test theory and method to do further judgment.

(3) Quality inspection of products. For example, the city quality inspection bureau does a quality of survey of gold sales products after received complaint. If the gold content of the necklace are subject to normal distribution, the test results can determine the quality of the product to sell the existence of the problem, but need to use hypothesis testing ideas and methods to make judgments.

(4) Factor effect problem. Consider, for example, the three different forms of advertising and five different prices on a commodity sales impact, selected from 15 large supermarket, every supermarket selection in a combination, the statistics of sales of a month, judge:

- Is there a significant difference in the sales of goods in different forms of advertising?

- Whether the difference in the sales of goods at different prices is significant?

The test of this significant problem requires the use of variance analysis in mathematical statistics.

\subsection{The application of multivariate statistics in commercial enterprises}

Multivariate statistical analysis is a statistical discipline which studies the relationship between multiple random variables and the intrinsic statistical regularity. At the same time, it can be classified and simplified by using different methods in multivariate statistical analysis. Multivariate statistical analysis includes: cluster analysis, discriminant analysis, principal component analysis, factor analysis, correspondence analysis, canonical correlation analysis, and so on.

Here, the focus of this paper is cluster analysis, discriminant analysis, principal component analysis, factor analysis method and its application in commercial enterprises.

\subsubsection{Cluster Analysis}

With the development of production technology and science, human understanding deepening, classification more and more finer, requirements increasingly higher, and alone the experience and professional knowledge is not the exact classification, we often need to quantitative and qualitative analysis combined to classification, so mathematical tools was gradually introduced into taxonomy, and format the numerical taxonomy. Later, with the introduction of multivariate analysis, cluster analysis is gradually separated from numerical taxonomy to form a relatively independent branch. Clustering analysis is very rich in content, there are systematic clustering method, ordered sample clustering, dynamic clustering, fuzzy clustering method, graph theory clustering method, clustering forecast method, etc.

\subsubsection{Discriminant Analysis}

In the production, scientific research and daily life, we often need to study the classification of objects based on the observed data. Discriminant analysis is to determine the type of the samples of a multivariate statistical analysis method. The purpose is to establish consists of a numerical index of classification rules on the classification of known data, and such rules are applied to the classification of unknown samples to classification. Discriminant analysis and cluster analysis are different. Discriminant analysis's content is very rich, and have a lot of methods. Discriminant analysis can ask questions from different angles, so it is different criteria, such as minimum Mahalanobis distance criterion, Fisher criterion, average loss minimum criterion, least square criterion, the maximum likelihood criterion, maximum likelihood criterion, and so on.

\subsubsection{Principal Component Analysis}

In practical problems, the study of multi index (variable) problem is often encountered, however, in most cases, there is a certain correlation between different indicators. As a result of many indicators, coupled with a certain correlation between indicators, is bound to increase the complexity of the analysis of the problem. Principal component analysis is try to recombine the original index into a new set of independent several comprehensive index to replace the original index, at the same time according to the actual needs of the desirable few comprehensive index, as far as possible to reflect 
the information of the original index. The statistical method that will be changed into a small number of mutually independent indicators, called principal component analysis.

\subsubsection{Factor Analysis}

The formation and development of factor analysis has a long history, and its earliest used to solve the problem of psychology and pedagogy research. Due to the large amount of calculation and lack of high-speed computing equipment, the application and development of factor analysis has greatly limits, even stagnate for a long time. Later, because of the appearance of the electronic computer, the theoretical research and calculation of factor analysis has been greatly progress. At present, the application of this method is very wide, and has made remarkable achievements in the fields of economics, sociology, and medicine and so on.

\section{Conclusion}

The application of statistical methods of enterprise must adhere to the principle to the facts as the basis and use the data to speak, to closely combine the application of statistical technology and professional technology. In consideration of statistical project implementation, we should from theory and fact level focus on analysis and conditions of use, carefully weigh the various related factors. In the promotion of scientific management today, both in the management decision-making and quality supervision, statistical methods should be given sufficient attention.

\section{References}

[1] Yu Xiulin, Ren cedar: Multivariate statistical analysis, China Statistics Press, 1999.8.

[2] Long Yonghong: Probability theory and mathematical statistics [M]. Higher Education Press, 2003.

[3] Yuan Yintang: Probability theory and mathematical statistics [M]. China Renmin University Press, 1990.

[4] Fu Zhiliang: Statistical methods of Analysis and diagnosis of enterprise product strength [j] Statistics and Decision Making, 2000(7). 